for more sets of exercises in the book, or for an accompanying exercise booklet, illustrating the rich field of applications of the ideas and listing general results. Another slight criticism that might be made of the overall effect of the book is that too many topics have been introduced into the 200 or so pages with the result that not enough space has been available for motivation and for applications. A professional pure mathematician will not of course feel this lack, but a beginning student or teacher wishing to bring his knowledge up to date may wish for more justification or illustrations at several places in the text.

J. HUNTER

\title{
Brief Mentions
}

LeITMan, G., Topics in Optimization (Academic Press Inc., New York, 1967), xv+ 469 pp., \$18.00.

This volume includes many results on the optimization of dynamical systems which extend or generalize the findings recorded in Optimization Techniques by the same editor and publisher in 1962. It is divided into two parts of five chapters each, the first describing results obtained by classical variational arguments and the second those involving modern geometrical concepts. The individual chapters coming from twelve authors (including one from Paris and two from Leningrad) are well written and the editor, who is also one of the contributors, has done a good job in producing a review of recent progress in a subject of increasing practical importance and theoretical interest.

KUSHNer, H. J., Stochastic Stability and Control (Academic Press Inc., New York, 1967), $x i+161 \mathrm{pp}$.

This monograph is devoted to the study of qualitative properties of Markov processes by the stochastic Liapunov function approach. The first two chapters treat respectively the necessary basic concepts from probability theory and stochastic stability. The remaining chapters are concerned with applications to the study of first exit times, of problems in optimal control, and of the design of optimal controls. It contains much original material and is well written so can be strongly recommended to applied mathematicians, statisticians and automatic control engineers.

TULlER, A., A Modern Introduction to Geometries (D. van Nostrand \& Co., London, 1967), ix+201 pp., 60s.

This compact book (suitable for undergraduates) illustrates the two principal approaches to geometry: the development of a body of theorems from a set of axioms and the study of the invariant theory of a transformation group. It is clearly written and attractively produced.

wallace, A. W., Differential Topology: First Steps (W. A. Benjamin Inc., New York, $1968), 130$ pp., $\$ 9.50$ cloth, $\$ 3.95$ paper.

Written for students with no previous knowledge of topology this small book is a useful introduction to certain aspects of the topology of manifolds which can be discussed without the use of advanced algebraic methods. The method of spherical modifications and the study of critical points of functions on manifolds are clearly presented. The emphasis throughout is on the geometric meaning of the ideas under consideration and on the motivation of the abstract concepts introduced. 
BENES, v. E., Mathematical Theory of Connecting Networks and Telephone Traffic (Academic Press Inc., New York, 1965), xiv+319 pp., \$14.00.

This book, which is a systematic study of communication networks, is an excellent illustration of the view that the content of modern applied mathematics is considerably more than the study of theoretical mechanics. It is concerned with three kinds of traffic theory: combinatorial problems of network design, probabilistic problems of traffic analysis and variational problems of routing traffic in networks.

Seminaire Bourbaki 1966-1967 (W. A. Benjamin Inc., New York), 314 pp., \$16.00.

In addition to reports of the 18 lectures presented at the Institut Henri Poincaré in the academic year 1966-67, this volume contains a cumulative name index and a complete table of contents by authors' names for this and the previous 13 volumes of the series (1948-1967).

PERvozvanskn, A. A., Random Processes in Nonlinear Control Theory (Academic Press Inc., New York, 1965), xii +342 pp., \$14.00.

This is a good translation (edited by I. Herzer) of the Russian edition of 1962 which has become recognized as a valuable source of problems and methods for nonlinear stochastic processes. It should prove extremely useful to anyone engaged in the study of either automatic control or stochastic processes.

Collatz, L., Functional Analysis and Numerical Mathematics (Academic Press Inc., New York, 1966), \$18.50.

This is an excellent translation (with some additional new material) of a work which has rapidly established itself as a modern classic. It makes an attractive. volume which should be in the hands of all applied mathematicians.

KURATowskI, K., Topology-Volume I (Academic Press Inc., New York, 1966), $\mathrm{xx}+560$ pp., $\$ 20.00$.

This book is a translation of the original French Topologie, $I$ published by P.W.N. in 1958 but the author has taken the opportunity to prepare a revised edition to which a great deal of new material has been added-for example, the first chapter has been increased from 63 pages to 150 pages. The printing is excellent and the book an essential part of the equipment of a modern pure mathematician.

SPOONER, G. A. AND MENTZER, R. L., Introduction to Number Systems (Prentice-Hall International, London, 1968), $x+339$ pp., 74s. 6d.

As indicated in the Preface, this book contains an introduction to number systems, together with the necessary basic language of sets, relations, mappings and operations, for college students whose primary interest is not mathematics.

CHREstenson, H. E., Mappings of the Plane (W. H. Freeman and Company Ltd, London, 1967), viii+171 pp., 18s.

The author determines the isometries (rigid motions) and similarities of a line, and, after an introduction to coordinates in a plane, the isometries and similarities of the plane. There are brief applications to plane trigonometry and complex numbers. Some of the work is presented in discovery form through the solving of successive exercise problems.

Mathematical Spectrum (Oxford University Press), Vol. 1, 1968-69, Number 1.

This is the first number of a new general interest magazine aimed at students of mathematics in schools, colleges and universities. It has been launched by the 
Applied Probability Trust, a non-profit making organisation established in 1963 with the support of the London Mathematical Society. The Editorial Board is drawn mainly from universities and there is an impressive international Advisory Board. It is intended that the magazine will contain elementary articles on any branch of mathematics and its applications, including educational matters and career opportunities, and will have a section devoted to problems. There is clearly a great need for more magazines of this type. This first number contains some interesting articles and some with nothing new or unusual. 